\title{
Ten years
} of Designed

\section{to Smile}

in

Wates

Aoife Nic lomhair, ${ }^{1}$ Mary Wilson ${ }^{2}$ and Maria Zeta

Morgan ${ }^{3}$ outline the structure of the DCP-led oral health programme Designed to Smile in Wales, and reflect on its success.

\section{Introduction}

Designed to Smile (D2S) is a national programme to improve the oral health of children in Wales. It is funded by the Welsh Government and delivered by the National Health Service, in partnership with nurseries, schools and other professional groups working towards a common aim: to prevent childhood dental disease. ${ }^{1}$ This school year (2019-20), the programme is celebrating its $10^{\text {th }}$ anniversary and the positive impact it has achieved.

\section{Author information}

'Dental Core Trainee, School of Dentistry, Belfast Health and Social Care Trust (and member of the BDJ Team reader panel); ${ }^{2}$ Consultant in Dental Public Health, Public Health Wales; ${ }^{3}$ Senior Lecturer in Dental Public Health, Cardiff University

\section{Background}

In the 2000s, with recognition of the widening gaps between the oral health of children from the most and least well-off families in Wales, the Welsh Government announced its commitment to tackling inequalities and to improving the oral health of children. ${ }^{2}$ At that time, over $50 \%$ of five-year-old children in Wales had experienced dental decay. ${ }^{2}$

The National Oral Health Action Plan for Wales (2007) included the development of a National Child Oral Health Improvement Programme, namely Designed to Smile, aimed at implementing sustainable toothbrushing programmes in school and nurseries, providing free toothbrushes and fluoride toothpaste to participating children' and additional fluoride varnish and fissure sealant programmes. ${ }^{2}$

In 2008, the D2S Programme was piloted in North and South East Wales, before being expanded to involve all Health Boards from September 2009. Responsibility for the implementation of D2S was allocated to the Community Dental Services, who organise and co-ordinate all aspects of the programme, with strategic support from Dental Public Health.

In light of emerging epidemiological ${ }^{3}$ and research evidence, ${ }^{4}$ and in order to ensure that the programme continued to target those children most at risk of dental decay, Welsh Government initiated a refocus of D2S in 2017. ${ }^{5}$ This involved realigning the programme to the 0 -5-year-old age-group, for a greater focus on the very youngest children with renewed collaboration with early years health professionals as part of the new Healthy Child Wales Programme. ${ }^{6}$

Structure and delivery of the current D2S programme

Designed to Smile involves:

1. A universal preventative programme for children from birth: this involves a wide range of professionals, including health visitors and other early years services. The aims are to help start good habits early by giving advice to families with young children, providing toothbrushes and 
toothpaste, and encouraging regular dental practice attendance

2. A targeted preventative programme for nursery and primary school children: this involves delivery of supervised toothbrushing and fluoride varnish programmes for children to help protect teeth against decay, and provision of oral health education resources. This element focuses on schools and nurseries within the three most deprived quintiles of the Welsh Index of Multiple Deprivation (WIMD). ${ }^{1}$

Table 1 shows the current D2S programme model across the different age groups and settings involved. ${ }^{1}$

During the 2018/19 academic year, 1,398 primary schools and nurseries, involving 90,977 children, participated in D2S daily toothbrushing schemes, while 44,217 of those children also had a fluoride varnish applied at school or nursery. ${ }^{7} 188,709$ toothbrushing home packs were distributed nationwide to encourage the development of effective preventative behaviours in the home setting. ${ }^{7}$

\section{Skill mix}

Employing staff from a diverse range of backgrounds and a plethora of previous working experiences, the $\mathrm{D} 2 \mathrm{~S}$ programme is an exemplar of skill mix in action. Table 2 shows the job role variety and distribution amongst D2S staff.?

Within dentistry, recent times have witnessed an increased focus on the development and utilisation of the extended skills of the whole dental team and on the concept of 'skill mix' to ensure the provision of services that are responsive to need, equitable and cost-effective. ${ }^{8,9}$ In contrast to the traditional view of the dentist as the main caregiver with a focus on intervention, 'skill-mix' describes a model of effective care, particularly prevention, being provided by other members of the dental team. ${ }^{8}$

Led and implemented by a diverse range of staff, including but not exclusively dental care professionals (DCPs) and with a focus on partnership working with teaching/childcare staff and health professionals (eg health visitors), D2S exemplifies the success that can be achieved through effective implementation of 'skill-mix' strategies, going beyond the traditional dental team.

\section{Flexible working model}

The D2S programme operates a flexible working model, aiming to accommodate the individual needs of staff and advocate a healthy work-life balance.

\section{Table 1 D2s Programme Model'}

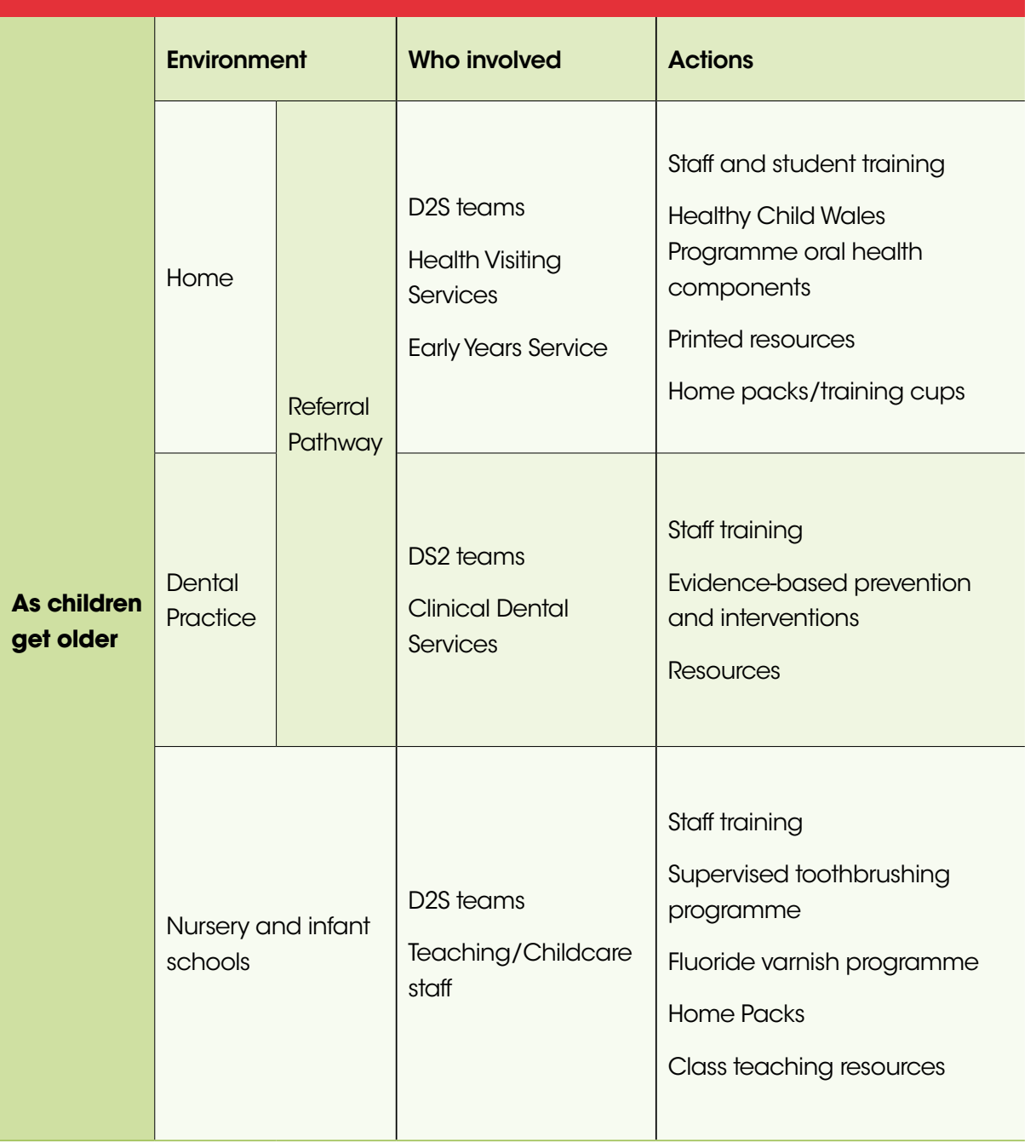

Table 2 Job role variety and distribution in the D2S programme ${ }^{7}$

\begin{tabular}{l|l} 
Role & \% of DS2 staff \\
\hline Administrator/ Driver & 7.2 \\
\hline Dental Health Support Worker & 31.6 \\
\hline Oral Health Educator/Promoter (Dental Nurse) & 46.7 \\
\hline Dental therapist/hygienist & 2.8 \\
\hline D2S Lead & 12.0 \\
\hline Senior Dental Officer & 0.6 \\
\hline WALES & $\mathbf{1 0 0}$ \\
\hline
\end{tabular}




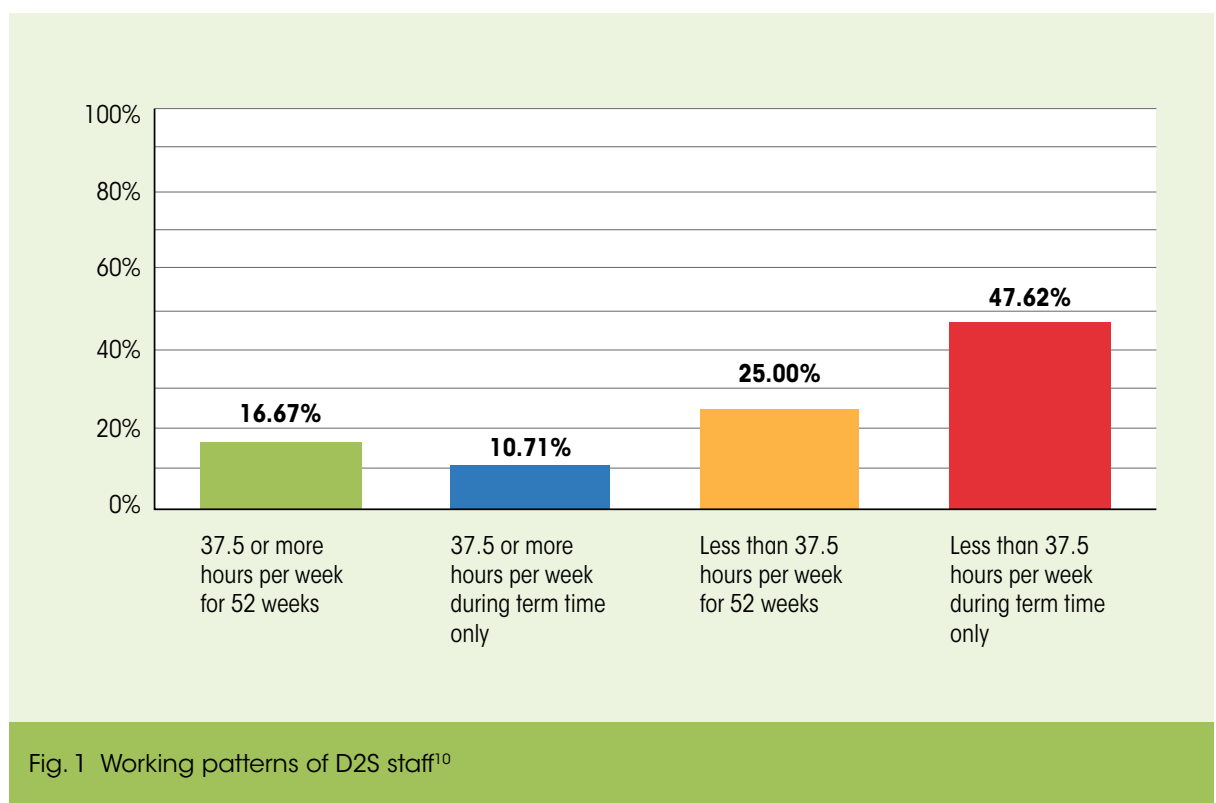

In a recent survey of D2S staff, respondents were asked to identify their individual employment contract arrangements (Fig. 1). The survey revealed that $50 \%$ of D2S staff fall between the ages of 25 and 44 years old, ${ }^{10}$ an age group in which there is likely to be high prevalence of employees with responsibility for young children.

With a large majority of staff working parttime hours and almost half working during term-time only, ${ }^{10}$ it is likely that the flexibility offered by the $\mathrm{D} 2 \mathrm{~S}$ programme is an attractive option to individuals with personal and family commitments, who wish to achieve a satisfactory balance between work and home life.

\section{Environmental sustainability}

The D2S programme promotes the concept of sustainable dentistry through its focus on the prevention of disease, subsequently reducing the need for restorative, extraction and sedation treatments with a higher carbon footprint. ${ }^{11}$ The delivery of the programme in community, nursery and school-based settings also reduces the carbon footprint associated with individual patient travel arrangements to dental surgery settings for equivalent procedures. ${ }^{11}$

In line with legislation requiring organisations both to calculate and understand their overall carbon footprints and in response to concerns regarding plastic waste resulting from the programme, an environmental impact assessment of D2S was recently carried out by Public Health Wales. ${ }^{12}$

This estimated that approximately 388 tonnes of carbon dioxide equivalents (CO2e) were attributable to the $\mathrm{D} 2 \mathrm{~S}$ programme in $2016 / 2017$ which, at $0.05 \%$ of the total carbon emissions produced by NHS activity in Wales could be considered a relatively minor contribution. ${ }^{12}$ However, acknowledging the need for continuous improvement, a number of initiatives have already been undertaken, including:

- Reducing paper use during administrative processes, while only utilising paper resourced from sustainable sources
- Changing the design concept for toothbrush storage in the classroom from the original 'brush bus' to a lighter 'tooth train' has reduced associated plastic by $70 \%$

- Promoting a recycling scheme for toothbrushes and toothpaste tubes with Terracycle UK among the settings involved in the programme, while providing advice regarding mainstream recycling of toothbrushes

Encouraging staff to consider their own travel behaviours and to adopt more sustainable modes of travel where possible, while making recommendations at an organisational level, such as car sharing schemes and use of the D2S Mobile Dental Units where available ${ }^{10}$

- Including sustainability as an important consideration in future tendering processes for new materials and products.
Impact of the D2S programme The most recent survey of five-year olds in Wales reported a reduction in the proportion of children with decay between 2007/08 (47.6\%) and 2015/16 (34.2\%). Although not possible to determine causality, this reduction in disease level coincided with the inception of the D2S programme in 2009 and was noted as constituting the 'first significant and sustained improvement in the levels of dental caries experienced by children in Wales since records began.' ${ }^{5}$

In 2007/08, 14 out of a class of 30 children would have decay experience, and these 14 children would have an average of 4.2 teeth affected. By 2015/16 this had fallen to ten children out of a class of 30 , and these 10 would have an average of 3.6 decayed teeth. ${ }^{13}$ The oral health of children in Wales improved across all social groups, with the most deprived WIMD quintile seeing the largest reduction in decay prevalence (by 15\%) and mean dmft score (by 0.6). ${ }^{13}$

The most recent survey of 12-year olds in Wales reported a $15 \%$ reduction in prevalence of dental decay from $45 \%$ in $2005 / 06$ to $30 \%$ in $2016 / 17 .^{14}$

\section{'The most recent survey of 12-year-olds}

\section{in Wales reported a $15 \%$ reduction in}

\section{prevalence of dental decay from $\mathbf{4 5 \%}$}

\section{in 2005/06 to $30 \%$ in 2016/17.'}

\section{Celebrating ten years!}

The 2019/2020 academic year marks ten years since the All-Wales introduction of the D2S programme. To celebrate this milestone, an Anniversary Week was held during September 2019. All participating nurseries, schools, dental practices and early years health services were encouraged to promote their involvement with D2S. The launch of the Twitter accounts (@D2S_Wales@CynllunGwen) enabled nurseries and schools to share their own events and festivities.

A national drawing competition to design a birthday card for Dewi, the D2S mascot, received an overwhelming response from nurseries and schools, with subsequent visits from Dewi to deliver prizes to the winning entrants across Wales (Fig. 2 and Fig. 3).

Schools and nurseries enrolled in the D2S programme at its commencement and 

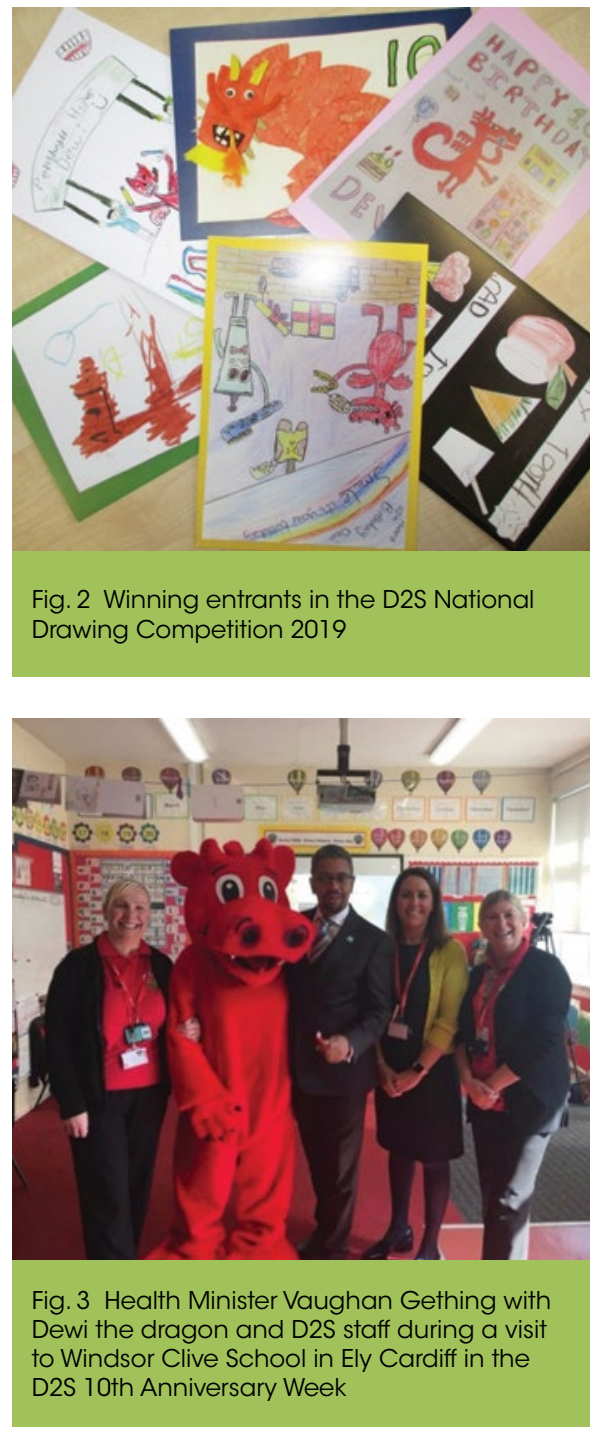

demonstrating outstanding commitment to D2S through sustained efforts in the ensuing years were also presented with plaques to commemorate the occasion and in recognition of their contribution to the improvements in oral health of Welsh children over the past ten years.

During the Anniversary Week, Health Minister Vaughan Gething and Chief Dental Officer, Colette Bridgman visited Windsor Clive School, in Ely, Cardiff to participate in an excellent toothbrushing programme in action.

Since these celebrations, the academic year of 2019/2020 has now developed to become one of two halves for the D2S programme as a result of the global outbreak of COVID-19, which began to emerge in the UK in early 2020. The subsequent closure of schools, nurseries and other services involved in the delivery of D2S has forced a stop to the preventative and educational activities central to the programme. Significant uncertainties exist at this time regarding the expected timescale of the outbreak, how daily life in the UK may look in the short to medium term and the potential implications on the $\mathrm{D} 2 \mathrm{~S}$ programme. It can be anticipated with certainty however, that significant challenges will have to be overcome.

\section{Conclusions}

The Well-being of Future Generations Act 2015 places responsibility on public bodies in Wales to work towards the achievement of seven 'Well-being goals' identified by the Act. ${ }^{15}$ These include: a prosperous Wales, a more resilient Wales, a more equal Wales, a healthier Wales, a Wales of cohesive communities, a Wales of vibrant culture and thriving Welsh language and a globally responsible Wales.

The D2S programme, through its emphasis on disease prevention, community-based healthcare, environmental sustainability and health equity makes very obvious strides towards the attainment of these goals.

Since the introduction of the programme a decade ago, significant improvements in the oral health of the most deprived children in Wales have been achieved, while the programme has been further refocused to ensure that it remains relevant and continues to direct intensive efforts and resources to the areas, families and children most in need, aligning with the principles of prudent healthcare.

The D2S programme also exemplifies the potential for successful utilisation of the extended skills of the whole dental team, along with others from non-clinical backgrounds in a flexible working model which advocates a heathy work-life balance.

Despite inevitable challenges to come, it is hoped that sustained efforts from the D2S programme in the next ten years will help to ensure that further reductions in the childhood levels of dental decay in Wales are realised, alongside associated reductions in the impact of dental pain, infection, poor nutrition, missed school days, general anaesthetics, dental anxiety and tooth loss on the next generation of Welsh children.

\section{References}

1. Designed to Smile website. Available at: http://www.designedtosmile.org/welcomecroeso/welcome/ (accessed 17 February 2020).

2. Welsh Government. WHC: Designed to Smile - A National Child Oral Health Improvement Programme Promoting Better Oral Health and Delivering a Fluoride Supplementation Programme. Welsh Government, 2008. Available at: http://www.wales.nhs.uk/documents/ WHC\%282008\%29008.pdf (accessed 17 February 2020).

3. Morgan M, Monaghan N. Picture of Oral Health 2015. Dental epidemiology survey of 3 year olds in Wales 2013-14. Cardiff
University/Public Health Wales, 2015.

4. Chestnutt I G, Chadwick B L, Hutchings S et al. Protocol for 'Seal or Varnish?' (SoV) trial: a randomised controlled trial to measure the relative cost and effectiveness of pit and fissure sealants and fluoride varnish in preventing dental decay. BMC Oral Health 2012; 12: 51.

5. Welsh Government. WHC: Re-focussing of the Designed to Smile child oral health improvement programme. Welsh Government, 2017. Available at: http:// www.designedtosmile.org/wp-content/ uploads/2017/09/WHC-D2S-refocusFINAL-16.06.17.pdf (accessed 17 February 2020).

6. Welsh Government. An overview of the Healthy Child Wales Programme. Welsh Government, 2016. Available at: https://gov.wales/sites/default/files/ publications/2019-05/an-overview-ofthe-healthy-child-wales-programme.pdf (accessed 17 February 2020).

7. Morgan M, Wilson M. Designed to Smile Monitoring report 2018-19. Cardiff University: Welsh Oral Health Information Unit, 2020.

8. Brocklehurst P, Macey R. Skill-mix in preventive dental practice - will it help address need in the future? BMC Oral Health 2015; 15(Suppl 1): S10.

9. BDJ Team. What does skill mix mean to your practice? BDJ Team 2015; 1: 15071. Available at: https://www.nature.com/articles/ bdjteam201571 (accessed 4 April 2020).

10. Nic Iomhair A. A survey of the commuting behaviours and influences of staff working in the Designed to Smile programme. Public Health Wales, 2019.

11. Mortimer F. The sustainable physician. Clin Med (Lond) 2010; 10: 110-112.

12. Bowden B. Evaluating the environmental impact of the Designed to Smile programme: Executive Summary. Public Health Wales, 2019.

13. Morgan M, Monaghan N. Picture of Oral Health 2018. Dental caries in 5-year olds 2015/6. Welsh Oral Health Information Unit: Cardiff University, 2017.

14. Morgan M, Monaghan N. Picture of Oral Health 2018. Dental epidemiological survey of 12-year olds 2016/17. Welsh Oral Health Information Unit: Cardiff University, 2018.

15. Welsh Government. Wellbeing of Future Generations (Wales) Act. Welsh Government, 2015. Available at: https:// futuregenerations.wales/about-us/futuregenerations-act/ (accessed 17 February 2020).

https://doi.org/10.1038/s41407-020-0285-1 